\title{
La acupuntura manual resultó más efectiva que la acupuntura simulada o la atención habitual en la profilaxis de la migraña sin aura
}

\author{
Manual acupuncture was more effective than sham acupuncture or routine care in preventing migraine without aura
}

\section{Comentado de:}

Xu S, y col. BMJ 2020;368:m697. PMID:322135091

\section{Objetivo}

Evaluar la eficacia de la acupuntura manual como tratamiento profiláctico para pacientes con migraña episódica sin aura.

\section{Diseño, lugar y participantes}

Ensayo clínico multicéntrico, aleatorizado y controlado, simple ciego (solo los participantes estaban cegados a la intervención). Realizado en siete hospitales de China, entre junio de 2016 y noviembre de 2018. Participaron 150 pacientes que no habían recibido nunca tratamiento con acupuntura, con migraña episódica sin aura.

\section{Intervención}

Se aleatorizó a los pacientes a recibir: a) 20 sesiones de acupuntura manual en puntos de acupuntura verdaderos más la atención habitual; b) 20 sesiones de acupuntura simulada no penetrante en puntos de no acupuntura heterosegmentales más la atención habitual; o c) atención habitual sola, durante ocho semanas.

\section{Medición de resultados principales}

Se evaluó el cambio en los días de migraña y los ataques de migraña cada cuatro semanas durante las semanas uno a 20 después de la asignación al azar, en comparación con el valor inicial (determinado cuatro semanas antes de la asignación al azar).

\section{Resultados}

Se aleatorizaron 150 pacientes con una media de edad de 36,5 años (Desvío Estándar [DE] 11,4); 123 [82\%] mujeres), de los cuales 147 fueron incluidos en el conjunto de análisis com- pleto. En comparación con la acupuntura simulada, la acupuntura manual obtuvo una reducción significativamente mayor en los días de migraña en las semanas 13 a 20, y una reducción significativamente mayor en los ataques de migraña en las semanas 17 a 20. La reducción en el número promedio de días de migraña fue 3,5 (DE 2,5) para la acupuntura manual versus 2,4 (DE $3,4)$ para la simulación (diferencia ajustada [DA] -1,4; Intervalo de Confianza [IC] 95\%) -2,4 a $-0,3$; p-valor=0,005) en las semanas 13 a 16; y 3,9 (DE 3,0) para la acupuntura manual versus 2,2 (DE 3,2) para la simulación (DA -2,1; IC 95\% -2,9 a $-1,2$; $p$-valor $<0,001$ ) en las semanas 17 a 20 . En las semanas 17 a 20 , la reducción en el número medio de ataques fue 2,3 (DE 1,7) para quienes recibieron acupuntura manual versus 1,6 (DE 2,5) para el grupo de acupuntura simulada (DA - 1,0; IC95\% $-1,5 \mathrm{a}-0,5 ; p<0,001)$. No se informaron eventos adversos graves. No se observaron diferencias significativas en la proporción de pacientes que perciben la penetración de la aguja entre la acupuntura manual y la acupuntura simulada ( $79 \%$ vs $75 \%$; p-valor= $0,891)$.

\section{Conclusiones}

Veinte sesiones de acupuntura manual fueron superiores a la acupuntura simulada y la atención habitual para la profilaxis de la migraña episódica sin aura. Estos resultados respaldan el uso de la acupuntura manual en pacientes que son reacios a usar medicamentos profilácticos o cuando estos medicamentos son ineficaces, y debe considerarse en futuras guías de práctica clínica. Fuente de financiamiento/Conflictos de interés de los autores: $E$ ensayo fue financiado por hospitales y universidades de China. Los autores declararon no tener relaciones financieras con ninguna organización que pudiera tener interés en est investigación u otras actividades o relaciones que pudieran haber influenciado este trabajo.

\section{Comentario}

La migraña es una de las causas más frecuentes de discapacidad en personas entre 15 y 49 años de edad ${ }^{2}$. Los expertos recomiendan medicación preventiva si la frecuencia de los síntomas es mayor a cuatro días al mes, pero solo alrededor de $13 \%$ de los pacientes acepta el tratamiento y muchos lo abandonan por sus efectos adversos o su ineficacia ${ }^{3,4}$, de ahí surge la importancia de encontrar alternativas para la profilaxis de la migraña mas allá de los tratamientos farmacológicos conocidos.

Aunque el estudio resumido fue de breve duración, los resultados mostraron que la administración de acupuntura redujo en mayor medida los días con migraña y los ataques de migraña, en comparación con la aplicación de acupuntura simulada. Se necesitan estudios con mayor periodo de seguimiento para determinar cuánto tiempo podrían durar los efectos beneficiosos de la acupuntura en los pacientes con este problema de salud. Asimismo, sería interesante conocer la eficacia de esta terapia frente a las alternativas de profilaxis farmacológica, evidencia que resultaría necesaria para incorporar a la acupuntura como tratamiento preventivo de primera línea. En la actualidad y en virtud de los resultados documentados, en la práctica se puede recomendar la acupuntura a los pacientes con migraña que no han respondido bien o que se resisten a la medicación preventiva.

Es importante destacar que la efectividad de la acupuntura puede ser variable de acuerdo al profesional que realiza su aplicación y a la técnica utilizada; además se debe tener en cuenta que la respuesta beneficiosa aparece en forma gradual.

\section{Conclusiones de la comentadora}

La acupuntura parece ser una herramienta eficaz para la prevención de la migraña sin aura, especialmente para las personas que prefieren tratamientos no farmacológicos, o para quienes que no toleraron o no tuvieron buena respuesta a la profilaxis 
farmacológica. Para que la acupuntura sea efectiva es importante que el médico acupunturista sea idóneo en el tema.

Ariana Fátima Cheng [ Medicina tradicional china, práctica privada. ariana.cheng@yahoo.com.ar ]

Cheng AF La acupuntura manual resultó más efectiva que la acupuntura simulada o la atención habitual en la profilaxis de la migraña sin aura. Evid Actual Pract Ambul. 2021;24(1):e002108. Comentado de: Xu S, et al. Manual acupuncture versus sham acupuncture and usual care for prophylaxis of episodic migraine without aura: multicentre, randomised clinical trial. BMJ. 2020;368:m697. PMID: 32213509

\section{Referencias}

1. Xu S, Yu L, Luo X, et al. Manual acupuncture versus sham acupuncture and usual care for prophylaxis of episodic migraine without aura: multicentre, randomised clinical trial. BMJ. 2020;368:697-697. Available from: 10.1136/bmj.m697.

2. Steiner TJ, Stovner LJ, Vos T, et al. Migraine is first cause of disability in under 50s: will health politicians now take notice? J Headache Pain. 2018;19(1). Available from: 10.1186/s10194-018-0846-2;https://dx.doi.org/10.1186/s10194-018-0846-2.

3. Lipton RB, Bigal ME, Diamond M, , et al. Migraine prevalence, disease burden, and the need for preventive therapy. Neurology. 2007;68(5):343-349. Available from: 10.1212/01.wnl.0000252808.97649.21.

4. Schulte LH, May A. Headache research in 2014: advancing migraine therapy. Lancet Neurol. 2015;14(1):6-7. Available from: 10.1016/s14744422(14)70295-9;https://dx.doi.org/10.1016/s1474-4422(14)70295-9. 